\title{
The Beta-Weibull Distribution on the Lattice of Integers
}

\author{
Vahid Nekoukhou, Hamid Bidram e Rasool Roozegar \\ Department of Statistics, Khansar Faculty of Mathematics and Computer Science, Khansar, Iran \\ v.nekoukhou@gmail.com \\ Department of Statistics, University of Isfahan, Isfahan, Iran \\ h.bidram@sci.ui.ac.ir \\ Department of Statistics, Yazd University, Yazd, Iran \\ rroozegar@yazd.ac.ir
}

\begin{abstract}
In this paper, a discrete analog of the beta-Weibull distribution is studied. This new distribution contains several discrete distributions as special sub-models. Some distributional and moment properties of the discrete beta-Weibull distribution as well as its order statistics are discussed. We will show that the hazard rate function of the new model can be increasing, decreasing, bathtub-shaped and upside-down bathtub. Estimation of the parameters is illustrated and the model with a real data set is also examined.
\end{abstract}

Keywords: Beta-Weibull distribution, Discrete beta generalized exponential distribution, Discrete Weibull distribution, Exponentiated discrete Weibull distribution, Hazard rate function. 


\section{Introduction}

Eugene et al. (2002) introduced a general class of distributions generated from the logit of a beta random variable with cumulative distribution function (cdf)

$$
F(x ; a, b, \boldsymbol{\theta})=I_{G(x)}(a, b)=\frac{1}{B(a, b)} \int_{0}^{G(x)} t^{a-1}(1-t)^{b-1} d t, x \in \mathbb{R},
$$

where $a>0$ and $b>0$ are two parameters whose role is to control the skewness and tail weight, $\boldsymbol{\theta}$ is the parameter vector of the absolutely continuous cdf $G, I_{y}(a, b)=\frac{B_{y}(a, b)}{B(a, b)}$ is the incomplete beta function ratio and $B_{y}(a, b)=\int_{0}^{y} t^{a-1}(1-t)^{b-1} d t$ denotes the incomplete beta function. In recent years, the class of beta- $G$ (BG) distributions has received much attention in the literature. For instance, Cordeiro et al. (2013a) and Singla et al. (2012) considered the beta exponentiated (generalized) Weibull distribution and investigated its properties and application. Cordeiro and Lemonte (2011a) and (2011b) studied the beta Laplace and beta-half-Cauchy distributions, respectively. Morais et al. (2013) considered the beta generalized logistic distribution and investigated some of its important properties and features. Cordeiro et al. (2013b) introduced the beta generalized Rayleigh distribution and considered its applications to lifetime data. Cordeiro et al. (2013c) and Bidram et al. (2013) introduced the beta Weibull-geometric distribution. Beta generalized Pareto distribution of Mahmoudi (2011), beta Lomax distribution of Lemonte and Cordeiro (2013), beta modified Weibull distribution of Silva et al. (2010) and beta log-normal distribution of Castellares et al. (2013) are other researches in this regard.

Recently, constructing discrete counterparts of known continuous distributions in order to study new discrete distributions has received much attention in the literature. Lisman and van Zuylen (1972) proposed and Kemp (1997) studied the discrete normal distribution which is characterized by maximum entropy for specified mean and variance. Roy (2003) introduced another discrete analog of normal distribution. Inusah and Kozubowski (2006) and Kozubowski and Inusah (2006) introduced Laplace and skew-Laplace distributions on the lattice of integers, respectively. Krishna and Pundir (2007) introduced discrete Maxwell distribution. Krishna and Pundir (2009) introduced discrete Burr distribution and studied a special case of the distribution which led to perform the discrete Pareto distribution. Gómez-Déniz and Calderin-Ojeda (2011) considered the discrete Lindley distribution and investigated some properties and applications of the model. Chakraborty and Chakravarty (2012) studied discrete gamma distributions and discussed estimation of the parameters. Nekoukhou et al. (2013a) studied the discrete beta exponential (DBE) distribution and illustrated that the hazard rate function of this discrete analogue of the beta exponential distribution of Nadarajah and Kotz (2006) is monotone. Moreover, Hussain and Ahmad (2014) and Chakraborty and Chakravarty (2014) introduced the discrete 
sain and Ahmad (2014) and Chakraborty and Chakravarty (2014) introduced the discrete inverse Rayleigh and discrete Gumbel distributions, respectively. Specially, Nekoukhou and Bidram (2015a) introduced a class of discrete BG distributions on the set of integers $\mathbb{Z}=\{0, \pm 1, \pm 2, \ldots\}$ emerges as

$$
f(y ; a, b, \boldsymbol{\theta})=P(Y=y)=I_{G(y+1)}(a, b)-I_{G(y)}(a, b) .
$$

The last authors investigated that the above probability mass function (pmf) can be expressed as

$$
f(y ; a, b, \boldsymbol{\theta})=\sum_{i=0}^{\infty} \omega_{i}(a, b)\left[\{G(y+1)\}^{a+i}-\{G(y)\}^{a+i}\right],
$$

where

$$
\omega_{i}(a, b)=\frac{\Gamma(a+b)(-1)^{i}}{\Gamma(a) \Gamma(b-i)(a+i) i !} .
$$

The cdf of a discrete BG distribution is also given by

$$
F(y ; a, b, \boldsymbol{\theta})=\sum_{i=0}^{\infty} \omega_{i}(a, b)\{G(\lfloor y\rfloor+1)\}^{a+i}, \quad y \in \mathbb{R},
$$

where $\lfloor$.$\rfloor denotes the integer function.$

Nekoukhou and Bidram (2015a) introduced the discrete beta generalized exponential (DBGE) distribution by inserting the cdf of the generalized exponential (GE) distribution of Gupta and Kundu (1999) into Equation (2). Indeed, they studied a discrete analog of the beta generalized exponential distribution of Barreto-Souza et al. (2010).

Here, we attempt to introduce a discrete analog of the beta-Weibull (BW) distribution as an another member of the discrete BG family of distributions. This is so because discrete random variables can also be encountered frequently for many different practical reasons. For example, in lifetime modeling, field failures are often collected and reported daily, weekly, and so forth. Systems often operate in cycles and the experimenter observes the number of cycles successfully completed prior to failure (cf. Lee and Cha, 2015).

We will see that an important characteristic of the new discrete distribution is that its hazard rate function can be decreasing, increasing, bathtub-shaped and upside-down bathtub depending on its parameters values. Hence, this discrete analog of the betaWeibull distribution can be considered as a flexible model in practical problems. In addition, the new discrete model contains several discrete distributions as special submodels.

Rest of the paper is organized as follows: Section 2, introduces the discrete beta-Weibull (DBW) distribution. Some important features and properties of the new distribution such as the probability mass and cumulative distribution functions are discussed. In addition, 
the mean and variance of the new model are illustrated. We consider the behavior of the hazard rate function and show that the new distribution exhibits bathtub, upsidedown bathtub, and monotonically increasing or decreasing hazard rates. Some special sub-models of the new distribution are given and the infinite divisibility of the distribuion in question is discussed. The cdf and pmf of the order statistics of DBW distributions are obtained. The estimation process of the parameters is discussed and a kind of simulated example is provided. Additionally, the stress-strength parameter is illustrated and the new model with a real data set is also examined. Finally, some concluding remarks are given in Section 3.

\section{The DHW distribution and its properties}

Here, we construct a discrete analog of the BW distribution by inserting the following cdf of the Weibull distribution,

$$
F(y ; \alpha, \beta)=1-e^{-\beta y^{\alpha}}, \quad y>0,
$$

where $\alpha>0$ and $\beta>0$ are the shape and scale parameters, respectively, into Equation (2). Consequently, the pmf of a DBW distribution for $y \in \mathbb{N}_{0}=\{0,1,2, \ldots\}$ is given by

$$
\begin{aligned}
f_{D B W}(y ; a, b, \alpha, p) & =\sum_{i=0}^{\infty} \omega_{i}(a, b)\left\{\left[1-e^{-\beta(y+1)^{\alpha}}\right]^{a+i}-\left[1-e^{-\beta y^{\alpha}}\right]^{a+i}\right\} \\
& =\sum_{i=0}^{\infty} \omega_{i}(a, b)\left\{\left(1-p^{(y+1)^{\alpha}}\right)^{a+i}-\left(1-p^{y^{\alpha}}\right)^{a+i}\right\}
\end{aligned}
$$

where $a>0, b>0, \alpha>0$ and $0<p=e^{-\beta}<1$ are the model parameters.

For integer values of $b$, the sum in Equation (5) is finite and stops at $b-1$; see Nekoukhou and Bidram (2015a).

The pmf given by Equation (5) defines the DBW distribution and it will be denoted by $\operatorname{DBW}(a, b, \alpha, p)$ in the rest of the paper.

It is interesting to note that the pmf of a DBW distribution is a linear combination of the pmf of three-parameter exponentiated discrete Weibull (EDW) probability mass functions, introduced by Nekoukhou and Bidram (2015b). An $\operatorname{EDW}(p, \alpha, \gamma)$ distribution with pmf

$$
\begin{aligned}
f_{E D W}(y ; p, \alpha, \gamma) & =\left\{1-p^{(y+1)^{\alpha}}\right\}^{\gamma}-\left\{1-p^{y^{\alpha}}\right\}^{\gamma} \\
& =\sum_{j=1}^{\infty}(-1)^{j+1}\left(\begin{array}{l}
\gamma \\
j
\end{array}\right)\left\{p^{j y^{\alpha}}-p^{j(y+1)^{\alpha}}\right\}
\end{aligned}
$$

where $\left(\begin{array}{l}\gamma \\ j\end{array}\right)=\frac{\Gamma(\gamma+1)}{\Gamma(\gamma+1-j) j !}$, is indeed a generalization of the discrete Weibull (DW) distribution of Nakagawa and Osaki (1975). For integer $\gamma>0$, the sum in Eq. (7) stops at $\gamma$. Therefore, we have the following representation for the pmf of a DBW distribution 


$$
f_{D B W}(y ; a, b, \alpha, p)=\sum_{i=0}^{\infty} \omega_{i}(a, b) f_{E D W}(y ; p, \alpha, a+i) .
$$

The pmf of the DBW distribution can also be written as a linear combination of the DW pmfs as follows

$$
f_{D B W}(y ; a, b, \alpha, p)=\sum_{i=0}^{\infty} \sum_{j=1}^{\infty} \omega_{i}(a, b)(-1)^{j+1}\left(\begin{array}{c}
a+i \\
j
\end{array}\right)\left\{p^{j y^{\alpha}}-p^{j(y+1)^{\alpha}}\right\} .
$$

For integer values of $a, \sum_{j=1}^{\infty}$ should be replaced by $\sum_{j=1}^{a+i}$ in the above equation. Figure 1 indicates the behavior of DBW pmfs for some values of their parameters.

Using Equation (3), the cdf of a DBW distribution is

$$
F_{D B W}(y ; a, b, \alpha, p)=\sum_{i=0}^{\infty} \omega_{i}(a, b) F_{E D W}(y ; p, \alpha, a+i), \quad y \geq 0,
$$

where $F_{E D W}$ denotes the cdf of $\operatorname{EDW}(p, \alpha, a+i)$ distributions. Hence, we have

$$
F_{D B W}(y ; a, b, \alpha, p)=\sum_{i=0}^{\infty} \omega_{i}(a, b)\left(1-p^{(\lfloor y\rfloor+1)^{\alpha}}\right)^{a+i}, \quad y \geq 0 .
$$

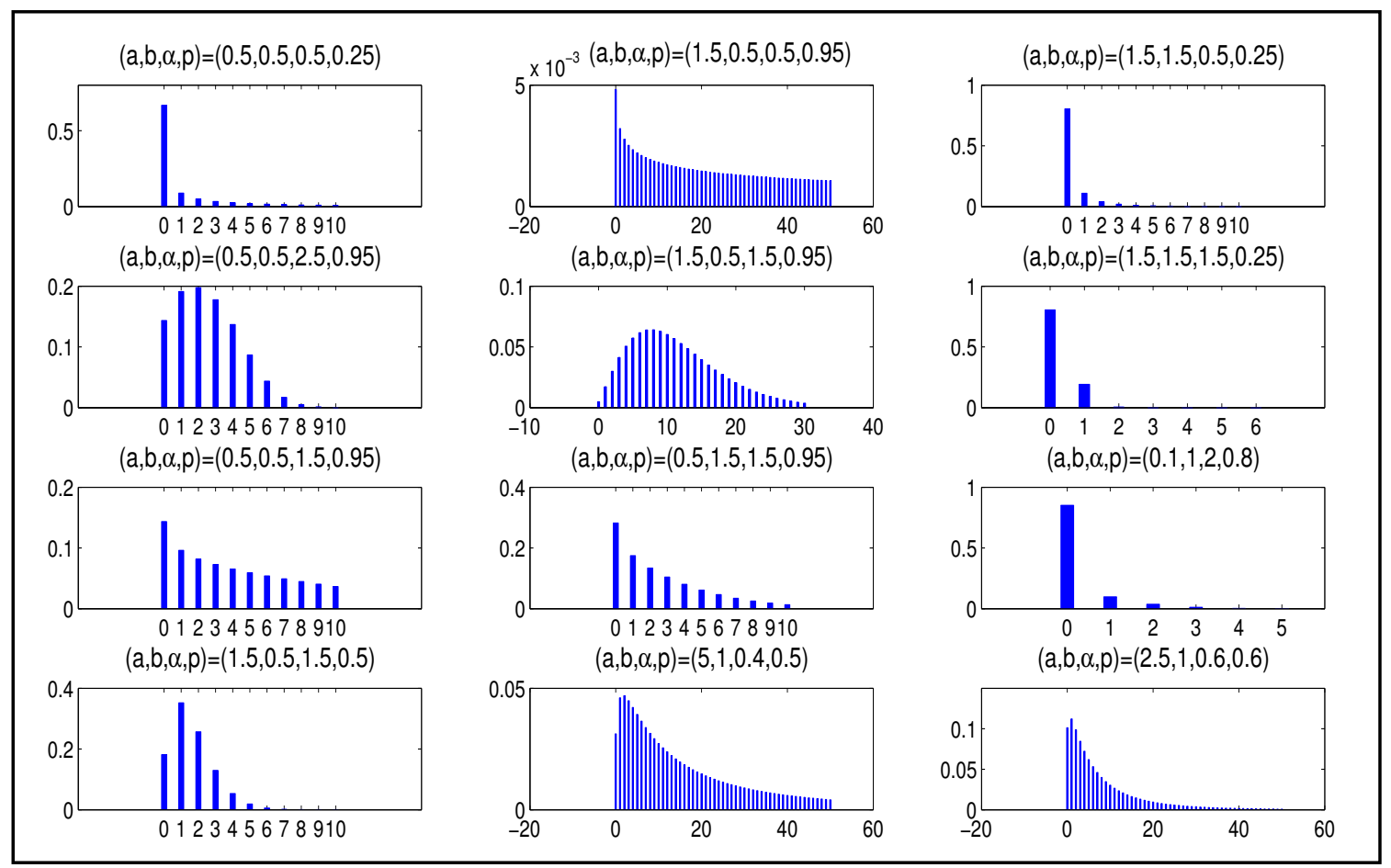

Figure 1: Pmfs of DBW distributions for some selected parameters values.

It is interesting to note that the probability density function of a BW distribution, in the continuous case, is also a linear combination of the exponentiated Weibull (EW) density functions introduced by Mudholkar et al. (1995).

The survival and hazard rate functions of a random variable $Y$ following a $\operatorname{DBW}(a, b, \alpha, p)$ distribution are given, respectively, by 


$$
\bar{F}_{D B W}(y ; a, b, \alpha, p)=1-\sum_{i=0}^{\infty} \omega_{i}(a, b)\left(1-p^{(\lfloor y\rfloor+1)^{\alpha}}\right)^{a+i}, \quad y \geq 0
$$

and

$$
h_{D B W}(y ; a, b, \alpha, p)=\frac{\sum_{i=0}^{\infty} \omega_{i}(a, b)\left\{\left(1-p^{(y+1)^{\alpha}}\right)^{a+i}-\left(1-p^{y^{\alpha}}\right)^{a+i}\right\}}{1-\sum_{i=0}^{\infty} \omega_{i}(a, b)\left(1-p^{(y+1)^{\alpha}}\right)^{a+i}}, \quad y \in \mathbb{N}_{0} .
$$

As mentioned before, for an integer $b>0, \sum_{i=0}^{\infty}$ should be replaced by $\sum_{i=0}^{b-1}$ in the above relations. Figure 2 illustrates the hazard rate function behavior of DBW distribution for different values of its parameters. As we see from Figure 2, the hazard rate function can be decreasing, increasing, bathtub-shaped and upside-down bathtub depending on its parameters values. The mean and variance of a random variable $Y \sim$ $\operatorname{DBW}(a, b, \alpha, p)$ are given by

$$
E(Y)=\sum_{i=0}^{\infty} \sum_{j=1}^{\infty} \sum_{y=0}^{\infty} \omega_{i}(a, b)(-1)^{j+1}\left(\begin{array}{c}
a+i \\
j
\end{array}\right) p^{j(y+1)^{\alpha}}
$$

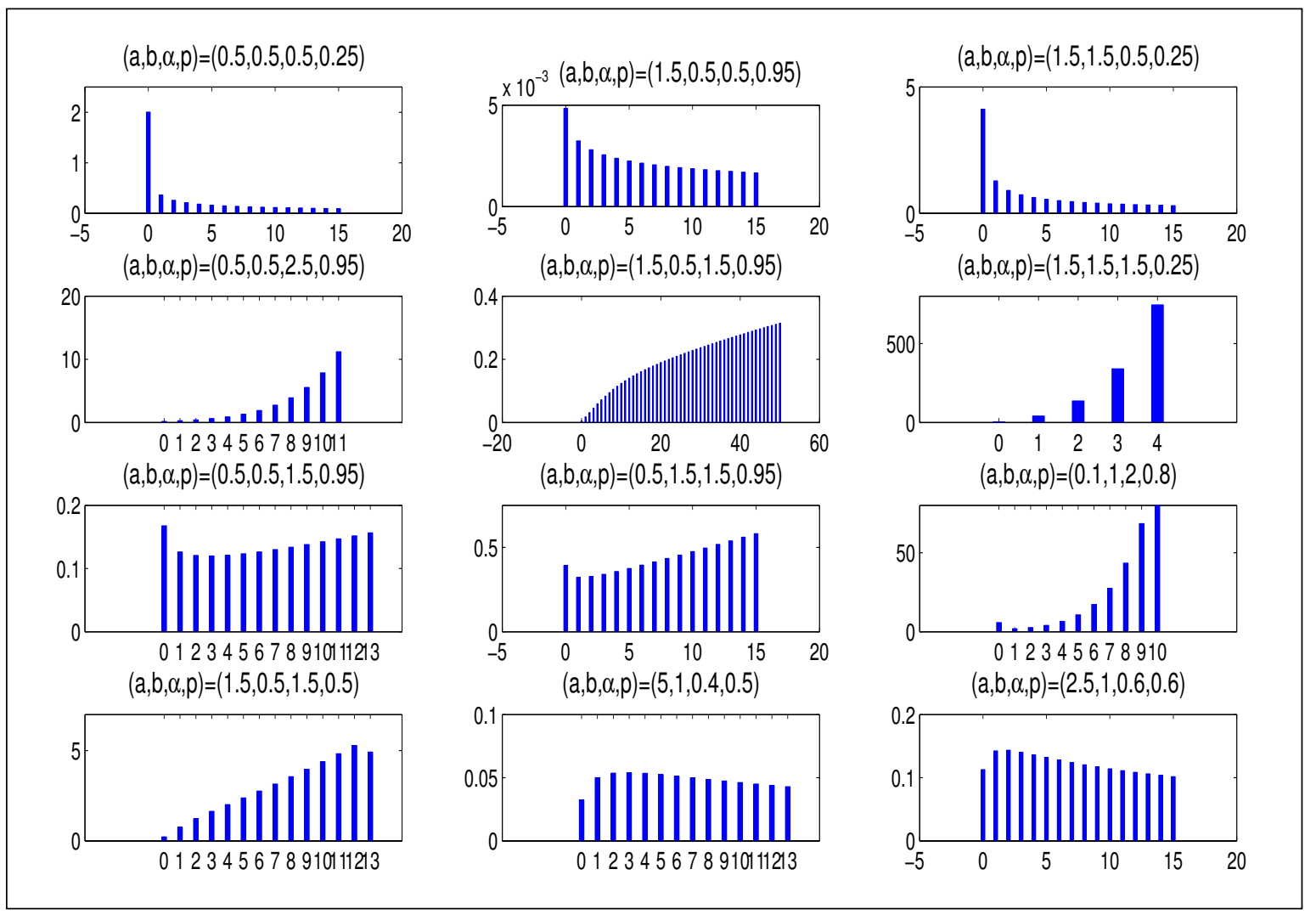

Figure 2: Hazard rate function plots of DBW distributions for selected parameters values.

and

$$
\operatorname{Var}(Y)=2 \sum_{i=0}^{\infty} \sum_{j=1}^{\infty} \sum_{y=0}^{\infty} \omega_{i}(a, b)(-1)^{j+1}\left(\begin{array}{c}
a+i \\
j
\end{array}\right) y p^{j(y+1)^{\alpha}}-\{E(Y)\}^{2}
$$


respectively.

The mean and variance of a DBW distribution for different values of its parameters are calculated in Table 1. It appears that for fixed values of $a$ and $b$, the mean increases (decreases) with $p(\alpha)$. In addition, DBW models are appropriate for modeling both overand under-dispersed data since, in these models, the variance can be larger or smaller than the mean which is not the case with some standard classical discrete distributions. Hence, the parameters of the underlying distribution can be adjusted to suit most data sets.

\subsection{Some important sub-models}

In this section, we consider some special cases of DBW distributions which are interesting and useful for their own sake.

Table 1: Mean (Variance) of $\operatorname{DBW}(a, b, \alpha, p)$ for different values of parameters.

\begin{tabular}{|c|c|c|c|c|c|c|}
\hline & \multicolumn{3}{|c|}{$(\mathrm{a}, \mathrm{b})=(0.5,0.5)$} & \multicolumn{3}{|c|}{$(\mathrm{a}, \mathrm{b})=(1,0.5)$} \\
\hline$\alpha / \mathrm{p}$ & 0.25 & 0.5 & 0.75 & 0.25 & 0.5 & 0.75 \\
\hline 0.25 & $23.188(8.362)$ & $52.462(2.195)$ & $62.282(2.792)$ & $36.225(1.265)$ & $79.860(3.173)$ & $82.865(3.666)$ \\
\hline 0.75 & $0.992(4.770)$ & $2.921(31.963)$ & $10.203(3.399)$ & $1.527(6.653)$ & $4.436(43.432)$ & $15.317(4.563)$ \\
\hline 1.00 & $0.654(1.504)$ & $1.609(6.509)$ & $4.390(39.165)$ & $1.000(2.000)$ & $2.414(8.243)$ & $6.464(48.249)$ \\
\hline 1.50 & $0.443(0.513)$ & 0.909 (1.396) & $1.950(4.703)$ & $0.672(0.638)$ & $1.339(1.586)$ & $2.792(5.041)$ \\
\hline 2.00 & $0.374(0.319)$ & $0.692(0.663)$ & $1.309(1.629)$ & $0.564(0.379)$ & $1.005(0.696)$ & $1.837(1.573)$ \\
\hline \multicolumn{4}{|c|}{$(\mathrm{a}, \mathrm{b})=(0.5,1)$} & \multicolumn{3}{|c|}{$(\mathrm{a}, \mathrm{b})=(1,1)$} \\
\hline$\alpha / \mathrm{p}$ & 0.25 & 0.5 & 0.75 & 0.25 & 0.5 & 0.75 \\
\hline 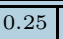 & $2.825(551.06)$ & $18.896(6770.39)$ & (51.307 (21750.07) & 5.565(1084.30) & $36.225(12651.13)$ & $886.221(34959.01)$ \\
\hline 0.75 & $0.225(0.524)$ & $0.832(3.964)$ & $3.275(44.252)$ & $0.430(0.936)$ & $1.527(6.654)$ & $5.807(71.559)$ \\
\hline 1.00 & $0.176(0.257)$ & $0.555(1.278)$ & $1.744(80.208)$ & $0.33(0.44)$ & $1.00(2.00)$ & $3.00(12.00)$ \\
\hline 1.50 & $0.144(0.145)$ & $0.382(0.451)$ & $0.955(1.615)$ & $0.271(0.240)$ & $0.672(0.638)$ & $1.579(2.025)$ \\
\hline 2.00 & $0.136(0.121)$ & $0.326(0.287)$ & $0.717(0.736)$ & $0.254(0.197)$ & $0.564(0.379)$ & $1.152(0.824)$ \\
\hline \multicolumn{4}{|c|}{$(a, b)=(1,1.5)$} & \multicolumn{3}{|c|}{$(\mathrm{a}, \mathrm{b})=(1.5,1)$} \\
\hline$\alpha / \mathrm{p}$ & 0.25 & 0.5 & 0.75 & 0.25 & 0.5 & 0.75 \\
\hline 0.25 & $1.137(92.564)$ & $13.999(3849.40)$ & $688.405(26493.12)$ & $8.225(1601.25)$ & $52.170(17799.51)$ & $109.152(43419.63)$ \\
\hline 0.75 & $0.168(0.266)$ & $0.755(2.152)$ & $3.206(24.087)$ & $0.615(1.263)$ & $2.117(8.555)$ & $7.849(90.211)$ \\
\hline 1.00 & $0.143(0.163)$ & $0.547(0.846)$ & $1.853(5.288)$ & $0.474(0.579)$ & $1.364(2.417)$ & 3.955 (13.994) \\
\hline 1.50 & $0.128(0.117)$ & $0.411(0.367)$ & $1.093(1.191)$ & $0.3813(0.230)$ & $0.897(0.703)$ & $2.014(2.098)$ \\
\hline 2.00 & $0.125(0.110)$ & $0.369(0.264)$ & $0.849(0.573)$ & $0.356(0.241)$ & $0.742(0.388)$ & $1.436(0.784)$ \\
\hline \multicolumn{4}{|c|}{$(\mathrm{a}, \mathrm{b})=(2,3)$} & \multicolumn{3}{|c|}{$(\mathrm{a}, \mathrm{b})=(3,2)$} \\
\hline$\alpha / \mathrm{p}$ & 0.25 & 0.5 & 0.75 & 0.25 & 0.5 & 0.75 \\
\hline 0.25 & $0.144(1.469)$ & $3.954(329.859)$ & $60.163(19166.1)$ & $1.599(59.660)$ & $25.245(5336.32)$ & $143.491(48960.12)$ \\
\hline 0.75 & $0.054(0.060)$ & $0.450(0.664)$ & $2.347(7.463)$ & $0.324(0.379)$ & $1.454(2.567)$ & $5.798(26.273)$ \\
\hline 1.00 & $0.052(0.051)$ & $0.371(0.370)$ & $1.530(2.163)$ & $0.285(0.253)$ & $1.057(0.973)$ & $3.265(5.207)$ \\
\hline 1.50 & $0.051(0.048)$ & $0.323(0.239)$ & $1.012(0.627)$ & $0.264(0.199)$ & $0.790(0.379)$ & $1.829(0.958)$ \\
\hline 2.00 & $0.051(0.048)$ & $0.313(0.217)$ & $0.836(0.336)$ & $0.262(0.193)$ & $0.709(0.249)$ & $1.358(0.395)$ \\
\hline
\end{tabular}

(1) If $b=1$, then the $\operatorname{DBW}(a, b, \alpha, p)$ distribution reduces to the $\operatorname{EDW}(p, \alpha, a)$ distribution of Nekoukhou and Bidram (2015b).

(2) If $a=b=1$, then the $\operatorname{DBW}(a, b, \alpha, p)$ distribution reduces to the $\mathrm{DW}(p, \alpha)$ distribution of Nakagawa and Osaki (1975).

(3) If $\alpha=b=1$, then the $\operatorname{DBW}(a, b, \alpha, p)$ distribution reduces to the discrete generalized exponential distribution of a second type, $\operatorname{DGE}_{2}(a, p)$ which was introduced by Nekoukhou et al. (2013). 
(4) If $a=b=\alpha=1$, then the geometric distribution is obtained from the $\operatorname{DBW}(a, b, \alpha, p)$ distribution.

(5) If $\alpha=1$, then the $\operatorname{DBW}(a, b, \alpha, p)$ distribution reduces to the discrete beta exponential distribution, $\operatorname{DBE}(a, b, p)$. This discrete version of the beta-exponential (BE) distribution, of Nadarajah and Kotz (2006), was introduced for the first time by Nekoukhou and Bidram (2015a) as a special case of the DBGE distribution. It must be mentioned that, recently, Nekoukhou et al. (2015) studied another discrete analog of the $\mathrm{BE}$ distribution using the relation

$$
P_{y}=\frac{f(y)}{\sum_{t=1}^{\infty} f(t)}, \quad y=1,2, \ldots
$$

where $f$ is the probability density function of the BE distribution. However, these two discrete analogs of the $\mathrm{BE}$ distribution have different structures.

(6) If $\alpha=2$ and $b=1$, then the $\operatorname{DBW}(a, b, \alpha, p)$ distribution reduces to the generalized discrete Rayleigh distribution, $\operatorname{GDR}(a, p)$, which introduced for the first time by Nekoukhou and Bidram (2015b).

(7) If $\alpha=2$ and $a=b=1$, then the discrete Rayleigh distribution of Roy (2004) is obtained.

(8) If $\alpha=2$, then the $\operatorname{DBW}(a, b, \alpha, p)$ distribution reduces to the discrete beta-Rayleigh distribution, $\operatorname{DBR}(a, b, p)$, studied by Nekoukhou (2015).

\subsection{Infinite divisibility}

Let us we make the following note in regards to the famous structural property of infinite divisibility of the new distribution in question. Such a characteristic has a close relation to the Central Limit Theorem and waiting time distributions. Thus, it is a desirable question in modeling to know whether a given distribution is infinitely divisible or not. To settle this question, we recall that according to Steutel and van Harn (2004, pp. 56), if $p_{x}, x \in \mathbb{N}_{0}$, is infinitely divisible, then $p_{x} \leq e^{-1}$ for all $x \in \mathbb{N}$. However, e.g., in a $\operatorname{DBW}(2.5,1.75,1.75,0.85)$ distribution we see that $p_{2}=0.371>e^{-1}=0.367$. Therefore, in general, $\operatorname{DBW}(a, b, \alpha, p)$ distributions are not infinitely divisible. In addition, since the classes of self-decomposable and stable distributions, in their discrete concepts, are subclasses of infinitely divisible distributions, we conclude that a DBW distribution can be neither self-decomposable nor stable in general.

\subsection{Order statistics}

Order statistics are the most fundamental tools in non-parametric statistics and inference. They enter the problems of estimation and hypothesis testing in a variety of ways. 
Nekoukhou and Bidram (2015a) obtained some general relations for the pmf and cdf of order statistics of the DBGE distribution. In the present section, we establish such relations for order statistics of the DBW distribution. More precisely, let $F_{i}(y ; a, b, \alpha, p)$ and $f_{i}(y ; a, b, \alpha, p)$ be the cdf and pmf of the $i$-th order statistic of a random sample of size $n$ drawn from a $\operatorname{DBW}(a, b, \alpha, p)$ distribution. One can show that the cdf of $i$-th order statistic is of the form

$$
F_{i}(y ; a, b, \alpha, p)=\sum_{k=i}^{n} \sum_{j=0}^{n-k} \sum_{m_{1}=0}^{\infty} \sum_{m_{2}=0}^{\infty} \ldots \sum_{m_{k+j}=0}^{\infty} \delta_{k, j} F_{E D W}\left(y ; p, \alpha, a(k+j)+\sum_{r=1}^{k+j} m_{r}\right),
$$

where

$$
\delta_{k, j}=\frac{\left(\begin{array}{c}
n \\
k
\end{array}\right)\left(\begin{array}{c}
n-k \\
j
\end{array}\right)(-1)^{j+\sum_{r=1}^{k+j} m_{r}}\{\Gamma(a+b)\}^{k+j}}{\{\Gamma(a)\}^{k+j} \prod_{r=1}^{k+j} \Gamma\left(b-m_{r}\right) m_{r} !\left(a+m_{r}\right)} .
$$

It should be noted that for integer $b>0$ the infinite sums in Equation (9) stop at $b-1$; see Nekoukhou and Bidram (2015a). As we see, the cdf of $i$-th order statistic of a $\operatorname{DBW}(a, b, \alpha, p)$ distribution is an infinite linear combination of $\operatorname{EDW}(p, \alpha, a(k+j)+$ $\left.\sum_{r=1}^{k+j} m_{r}\right)$ distribution functions. In particular, when $b=1$, one can show that Equation (9) reduces to the cdf of $i$-th order statistic of an $\operatorname{EDW}(p, \alpha, a)$ distribution.

The corresponding pmf of $i$-th order statistic, $f_{i}(y)=F_{i}(y)-F_{i}(y-1)$ for an integer value of $y$, is also given by

$$
f_{i}(y ; a, b, \alpha, p)=\sum_{k=i}^{n} \sum_{j=0}^{n-k} \sum_{m_{1}=0}^{\infty} \sum_{m_{2}=0}^{\infty} \ldots \sum_{m_{k+j}=0}^{\infty} \delta_{k, j} f_{E D W}\left(y ; p, \alpha, a(k+j)+\sum_{r=1}^{k+j} m_{r}\right),
$$

where $f_{E D W}$ denotes the pmf of an EDW distribution given by (6)-(7). In view of the fact that $f_{i}(y ; a, b, \alpha, p)$ is an infinite linear combination of $\operatorname{EDW}\left(p, \alpha, a(k+j)+\sum_{r=1}^{k+j} m_{r}\right)$ pmfs, we may obtain some properties of order statistics, such as their moments, from the corresponding EDW distributions.

\subsection{Estimation}

Let $Y$ be a random variable from the DBW distribution and $\boldsymbol{\theta}=(a, b, \alpha, p)^{T}$ be the parameters vector. The likelihood function for a single observation $y$ is given by

$$
\ell(\boldsymbol{\theta})=\sum_{i=0}^{\infty} \omega_{i}(a, b)\left\{\left(1-p^{(y+1)^{\alpha}}\right)^{a+i}-\left(1-p^{y^{\alpha}}\right)^{a+i}\right\} .
$$

The first derivatives of the likelihood function with respect to the components of $\boldsymbol{\theta}$ are given by

$$
\begin{aligned}
\frac{\partial \ell}{\partial a}=\quad \sum_{i=0}^{\infty} \quad \omega_{i}(a, b)\left(\psi(a+b)-\psi(a)-\frac{1}{a+i}\right)\left\{\left(1-p^{(y+1)^{\alpha}}\right)^{a+i}-\left(1-p^{y^{\alpha}}\right)^{a+i}\right\} \\
+\quad \omega_{i}(a, b)\left\{\left(1-p^{(y+1)^{\alpha}}\right)^{a+i} \ln \left(1-p^{(y+1)^{\alpha}}\right)-\left(1-p^{y^{\alpha}}\right)^{a+i} \ln \left(1-p^{y^{\alpha}}\right)\right\},
\end{aligned}
$$




$$
\begin{gathered}
\frac{\partial \ell}{\partial b}=\sum_{i=0}^{\infty} \omega_{i}(a, b)(\psi(a+b)-\psi(b-i))\left\{\left(1-p^{(y+1)^{\alpha}}\right)^{a+i}-\left(1-p^{y^{\alpha}}\right)^{a+i}\right\} \\
\frac{\partial \ell}{\partial \alpha}=\sum_{i=0}^{\infty} \quad \omega_{i}(a, b)(a+i) \ln p\left\{\left(1-p^{y^{\alpha}}\right)^{a+i-1} p^{y^{\alpha}} y^{\alpha} \ln y\right. \\
\left.-\quad\left(1-p^{(y+1)^{\alpha}}\right)^{a+i-1} p^{(y+1)^{\alpha}}(y+1)^{\alpha} \ln (y+1)\right\}
\end{gathered}
$$

and

$\frac{\partial \ell}{\partial p}=\sum_{i=0}^{\infty} \omega_{i}(a, b)(a+i)\left\{\left(1-p^{y^{\alpha}}\right)^{a+i-1} p^{y^{\alpha}-1} y^{\alpha}-\left(1-p^{(y+1)^{\alpha}}\right)^{a+i-1} p^{(y+1)^{\alpha}-1}(y+1)^{\alpha}\right\}$,

where $\psi($.$) is the well-known digamma function.$

Now, let $y_{1}, y_{2}, \ldots, y_{n}$ be $n$ observations of a random sample drawn from a $\operatorname{DBW}(a, b, \alpha, p)$ distribution. The total likelihood function is obtained by $\ell_{n}(\boldsymbol{\theta})=\prod_{k=1}^{n} \ell_{k}(\boldsymbol{\theta})$, where $\ell_{k}(\boldsymbol{\theta})$; $k=1,2, \ldots, n$, is given by Equation (10). The maximum likelihood estimate (MLE) of $\boldsymbol{\theta}$, say $\hat{\boldsymbol{\theta}}$, is obtained by solving the nonlinear equation

$$
\boldsymbol{U}_{n}=\left(\partial \ell_{n} / \partial a, \partial \ell_{n} / \partial b, \partial \ell_{n} / \partial \alpha, \partial \ell_{n} / \partial p\right)^{T}=\mathbf{0},
$$

using a numerical method.

The Fisher information matrix is given by

$$
\boldsymbol{I}(\boldsymbol{\theta})=\left[I_{\theta_{i}, \theta_{j}}\right]_{4 \times 4} ; \quad i, j=1,2,3,4,
$$

whose components can be calculated, numerically, by the relation

$$
I_{\theta_{i}, \theta_{j}}=E\left(-\frac{\partial^{2} \ell}{\partial \theta_{i} \partial \theta_{j}}\right) ; \quad i, j=1,2,3,4 \text {. }
$$

The total Fisher information matrix is given by $\boldsymbol{I}_{n}(\boldsymbol{\theta})=n \boldsymbol{I}(\boldsymbol{\theta})$ which can be approximated by

$$
\boldsymbol{I}_{n}(\hat{\boldsymbol{\theta}}) \approx\left[-\left.\frac{\partial^{2} \ell_{n}}{\partial \theta_{i} \partial \theta_{j}}\right|_{\boldsymbol{\theta}=\hat{\boldsymbol{\theta}}}\right]_{4 \times 4} \quad i, j=1,2,3,4
$$

and, therefore, under conditions given in Ferguson (1996), $\hat{\boldsymbol{\theta}}$ has an asymptotic normal distribution as $N_{4}\left(\boldsymbol{\theta}, \boldsymbol{I}_{n}(\hat{\boldsymbol{\theta}})^{-1}\right)$, or equivalently, $\boldsymbol{I}_{n}(\hat{\boldsymbol{\theta}})^{1 / 2}(\hat{\boldsymbol{\theta}}-\boldsymbol{\theta})^{T}$ has a multivariate standard normal distribution. Asymptotic normal distributions are usually used for constructing approximate confidence intervals, confidence regions, and testing hypotheses of the parameters. For example, an asymptotic confidence interval with confidence coefficient $1-\gamma$ for parameters $\theta_{j} ; j=1,2,3,4$, is computed by $\left(\hat{\theta}_{j} \pm z_{1-\gamma / 2} \sqrt{I_{\hat{\theta_{j}}, \hat{\theta_{j}}}}\right)$, where $I_{\hat{\theta}_{j}, \hat{\theta}_{j}}$ is the $j$ th diagonal element of $\boldsymbol{I}_{n}(\hat{\boldsymbol{\theta}})^{-1}$ and $z_{1-\gamma / 2}$ is $(1-\gamma / 2)$-th quantile of the standard normal distribution. 


\subsection{A simulated example}

It is evident that if $V$ is a beta random variable with parameters $a$ and $b$, then the cdf of the random variable $X=G^{-1}(V)$ coincides with cdf (1). Hence, for a Weibull random variable with cdf (4), we have

$$
X=\left(-\frac{1}{\beta} \log (1-V)\right)^{\frac{1}{\alpha}},
$$

which helps to generate data from a BW distribution. Thus, we can consider $\lfloor X\rfloor$ in order to generate data from a $\operatorname{DBW}(a, b, \alpha, p)$ distribution. Table 2 presents the maximum likelihood estimates of $\boldsymbol{\theta}=(a, b, \alpha, p)^{T}$ of a DBW distribution and also contains their standard errors for the sample sizes $n=100,200,500$ and 1000 as a simulated example. Standard errors are obtained by means of the asymptotic covariance matrix of the MLEs of DBW parameters when the Newton-Raphson procedure converges in, e.g., MATLAB software.

Table 2: MLEs and standard errors of the DBW parameters

\begin{tabular}{ccccccccc}
\hline$n$ & $\hat{a}$ & $\hat{b}$ & $\hat{\alpha}$ & $\hat{p}$ & $\hat{S E}(\hat{a})$ & $\hat{S E}(\hat{b})$ & $\hat{S E}(\hat{\alpha})$ & $\hat{S E}(\hat{p})$ \\
\hline$(a, b, \alpha, p)=(1,2,0.5,0.25)$ & & & & & & & & \\
\hline 25 & 0.8316 & 1.6723 & 0.2813 & 0.2022 & 0.8324 & 0.8716 & 1.5297 & 0.4879 \\
100 & 0.8623 & 1.6211 & 0.2987 & 0.1912 & 0.7898 & 0.6373 & 1.2382 & 0.2666 \\
200 & 0.8214 & 1.5732 & 0.4142 & 0.2323 & 0.5575 & 0.5161 & 0.9875 & 0.1252 \\
500 & 0.9550 & 1.8879 & 0.5465 & 0.2412 & 0.2242 & 0.2333 & 0.4979 & 0.0822 \\
1000 & 0.9773 & 1.9412 & 0.5150 & 0.2510 & 0.1012 & 0.1161 & 0.2242 & 0.0374 \\
\hline$(a, b, \alpha, p)=(2,3,1.5,0.5)$ & & & & & & & & \\
\hline 25 & 1.6404 & 2.7012 & 1.3145 & 0.4320 & 0.8954 & 0.7882 & 1.2122 & 0.4958 \\
100 & 1.8363 & 2.8474 & 1.1887 & 0.4447 & 0.7191 & 0.5464 & 0.9925 & 0.2112 \\
200 & 1.9612 & 2.8997 & 1.7012 & 0.4923 & 0.4362 & 0.2838 & 0.8131 & 0.1573 \\
500 & 2.0484 & 2.9721 & 1.4954 & 0.5102 & 0.2545 & 0.1828 & 0.5012 & 0.0685 \\
1000 & 1.9954 & 3.0050 & 1.5076 & 0.5101 & 0.1131 & 0.1124 & 0.2112 & 0.0207 \\
\hline 25 & & & & & & & & \\
\hline$(a, b, \alpha, p)=(2,3,0.75)$ & 1.7971 & 2.2631 & 2.6982 & 0.8175 & 0.7813 & 0.6993 & 0.9011 & 0.3984 \\
200 & 1.8892 & 2.3547 & 3.4455 & 0.7323 & 0.5373 & 0.3897 & 0.7725 & 0.2837 \\
500 & 1.9232 & 1.8099 & 2.9202 & 0.7042 & 0.2333 & 0.2259 & 0.5541 & 0.1276 \\
1000 & 1.9777 & 2.1176 & 3.1138 & 0.7507 & 0.1921 & 0.1611 & 0.2721 & 0.0784 \\
& 2.0074 & 2.1031 & 2.9973 & 0.7575 & 0.1043 & 0.1108 & 0.1112 & 0.0314 \\
\hline
\end{tabular}

Figure 3 is plotted for more values of sample sizes and for $(a, b, \alpha, p)=(1,2,0.5,0.25)$ to show how the four standard errors vary with respect to $n$. As we see, the standard errors for each parameter decrease to zero as $n \rightarrow \infty$. 


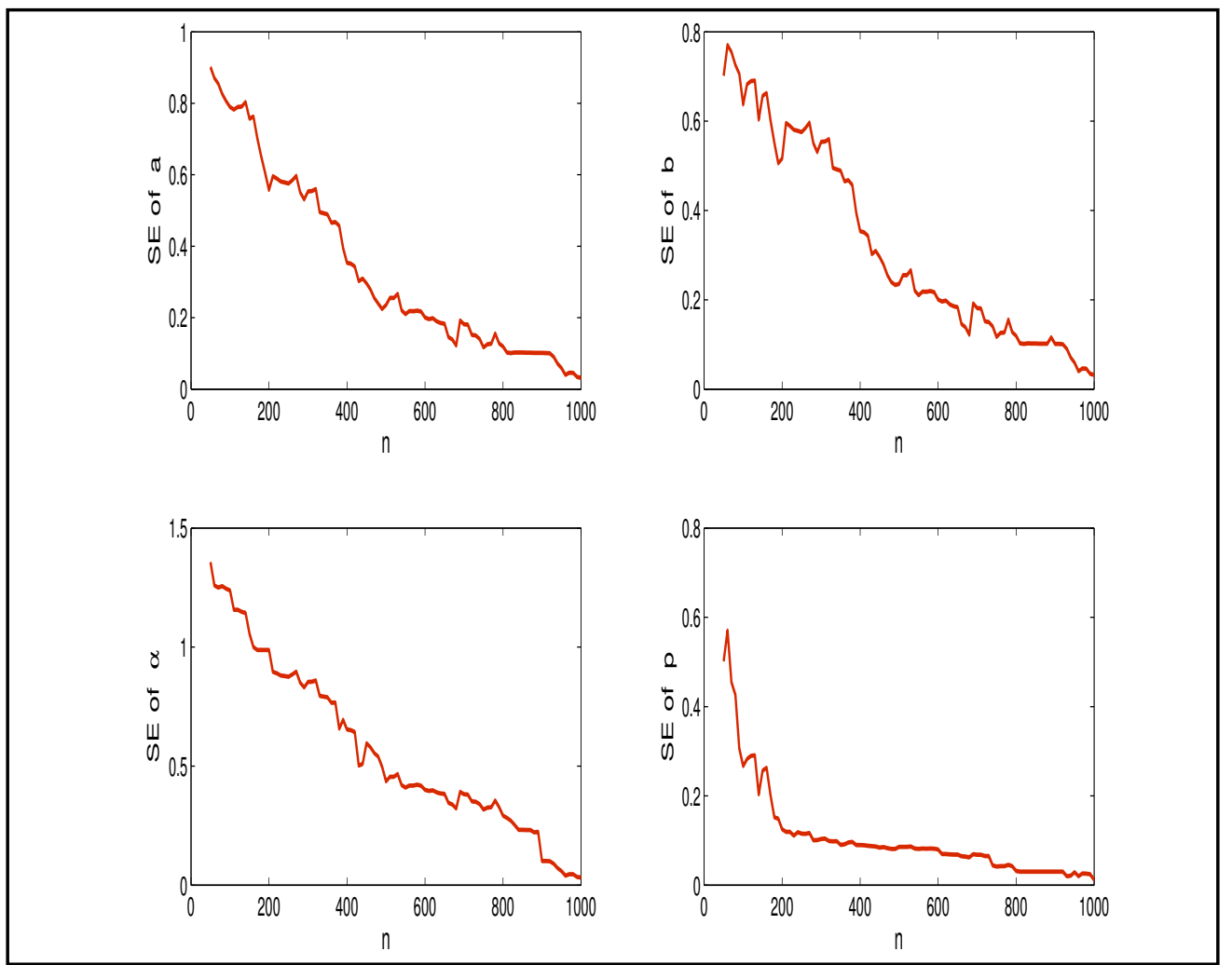

Figure 3: Standard errors of the MLEs of $(a, b, \alpha, p)=(1,2,0.5,0.25)$ for $\mathrm{n}=50,100,150, \ldots, 1000$.

\subsection{Stress-strength parameter}

The stress-strength parameter $R=P(X>Y)$ is a measure of component reliability and its estimation problem when $X$ and $Y$ are independent and follow a specified common distribution has been discussed widely in the literature. Suppose that the random variable $X$ is the strength of a component which is subjected to a random stress $Y$. Estimation of $R$ when $X$ and $Y$ are independent and identically distributed following a well-known distribution has been considered in the literature; see Kotz et al. (2003).

Sometimes, data may be discrete by nature, for example, the number of events occurring in a certain spatial or temporal interval. When discrete data are derived from discretization, instead of numerical measurements on $X$ and $Y$, they are presented in a form of ordered categories.

Here, we consider the estimation of $R$ in a DBW model, in view of the fact that a relatively small amount of work is devoted to discrete or categorical data.

The stress-strength parameter, in discrete case, is defined as

$$
R=P(X>Y)=\sum_{x=0}^{\infty} f_{X}(x) F_{Y}(x),
$$

where $f_{X}$ and $F_{Y}$ denote the pmf and cdf of the independent discrete random variables $X$ and $Y$, respectively. Now, let $X \sim \operatorname{DBW}\left(\boldsymbol{\theta}_{\mathbf{1}}\right)$ and $Y \sim \operatorname{DBW}\left(\boldsymbol{\theta}_{\mathbf{2}}\right)$, where $\boldsymbol{\theta}_{\mathbf{1}}=\left(a_{1}, b_{1}, \alpha, p\right)^{T}$ and $\boldsymbol{\theta}_{\mathbf{2}}=\left(a_{2}, b_{2}, \alpha, p\right)^{T}$. Using Equations (5) and (8), we obtain 
$R=\sum_{i=0}^{\infty} \sum_{j=0}^{\infty} w_{i}\left(a_{1}, b_{1}\right) w_{j}\left(a_{2}, b_{2}\right) \sum_{x=0}^{\infty}\left\{\left(1-p^{(x+1)^{\alpha}}\right)^{\left(a_{1}+i\right)}-\left(1-p^{x^{\alpha}}\right)^{\left(a_{1}+i\right)}\right\}\left(1-p^{(x+1)^{\alpha}}\right)^{\left(a_{2}+j\right)}$.

Now, assume that $x_{1}, x_{2}, \ldots, x_{n}$ and $y_{1}, y_{2}, \ldots, y_{m}$ are independent observations from $X \sim \operatorname{DBW}\left(\boldsymbol{\theta}_{\mathbf{1}}\right)$ and $Y \sim \operatorname{DBW}\left(\boldsymbol{\theta}_{\mathbf{2}}\right)$, respectively. The total likelihood function is $\ell_{R}\left(\boldsymbol{\theta}^{*}\right)=$ $\ell_{n}\left(\boldsymbol{\theta}_{\mathbf{1}}\right) \ell_{m}\left(\boldsymbol{\theta}_{\mathbf{2}}\right)$, where $\boldsymbol{\theta}^{*}=\left(\boldsymbol{\theta}_{\mathbf{1}}, \boldsymbol{\theta}_{\mathbf{2}}\right)$. The score vector is given by

$$
U_{R}\left(\boldsymbol{\theta}^{*}\right)=\left(\partial \ell_{R} / \partial a_{1}, \partial \ell_{R} / \partial a_{2}, \partial \ell_{R} / \partial b_{1}, \partial \ell_{R} / \partial b_{2}, \partial \ell_{R} / \partial \alpha, \partial \ell_{R} / \partial p\right),
$$

and the MLE of $\boldsymbol{\theta}^{*}$, say $\hat{\boldsymbol{\theta}}^{*}$, may be attained from the nonlinear equation $U_{R}\left(\hat{\boldsymbol{\theta}}^{*}\right)=\mathbf{0}$. Thus, by inserting the MLEs in Equation (11) the stress-strength parameter $R$ will be estimated.

\subsection{Application}

In this section, the DBW distribution will be examined for the fish catch data, given by Kemp (1992). These data have been displayed in Table 3. Gómez-Déniz (2010) used these data in order to study its generalization of the geometric distribution.

Table 3: Fish catch data

\begin{tabular}{cccccccccc}
\hline Numbers & 0 & 1 & 2 & 3 & 4 & 5 & 6 & 7 & 8 \\
\hline Frequency & 1 & 2 & 11 & 20 & 29 & 23 & 10 & 3 & 1 \\
\hline
\end{tabular}

Here, we compare the capacity of the DBW model with some of its rival models in discrete data modeling which are notified briefly in the sequence.

(1) Discrete Weibull distribution of Nakagawa and Osaki (1975), with pmf

$$
f(y ; p, \alpha)=\left(1-p^{(y+1)^{\alpha}}\right)-\left(1-p^{y^{\alpha}}\right)
$$

which is a special case of the EDW distribution, when $\gamma=1$.

(2) Discrete generalized exponential distribution of a second type, $\operatorname{DGE}_{2}(\gamma, p)$, of Nekoukhou et al. (2013) with pmf

$$
f(y ; p, \gamma)=\left\{1-p^{(y+1)}\right\}^{\gamma}-\left\{1-p^{y}\right\}^{\gamma},
$$

which can be considered as another special case of the EDW distribution, by choosing $\alpha=1$.

(3) If $\alpha=2$, then the pmf of $\operatorname{EDW}(p, \alpha, \gamma)$ distribution reduces to

$$
\begin{aligned}
f(y ; p, \gamma) & =\left\{1-p^{(y+1)^{2}}\right\}^{\gamma}-\left\{1-p^{y^{2}}\right\}^{\gamma} \\
& =\sum_{j=1}^{\infty}(-1)^{j+1}\left(\begin{array}{l}
\gamma \\
j
\end{array}\right) p^{j y^{2}}\left(1-p^{j(2 y+1)}\right),
\end{aligned}
$$


which defines the generalized discrete Rayleigh distribution, $\operatorname{GDR}(\gamma, p)$, studied by Nekoukhou and Bidran (2015b).

(4) The pmf of a discrete beta generalized exponential (DBGE) distribution proposed by Nekoukhou and Bidram (2015a), with parameters $a>0, b>0, \alpha>0$ and $0<p<1$, is of the form

$$
\begin{aligned}
f(y ; a, b, \alpha, p) & =\sum_{i=0}^{\infty} \omega_{i}(a, b)\left\{\left(1-p^{y+1}\right)^{\alpha(a+i)}-\left(1-p^{y}\right)^{\alpha(a+i)}\right\} \\
& =\sum_{i=0}^{\infty} \omega_{i}(a, b) \sum_{j=1}^{\infty}(-1)^{j+1}\left(\begin{array}{c}
\alpha(a+i) \\
j
\end{array}\right) p^{j y}\left(1-p^{j}\right),
\end{aligned}
$$

where $y \in \mathbb{N}_{0}=\{0,1,2, \ldots\}$. The above pmf is in fact a linear combination of the probability mass functions of the $\mathrm{DGE}_{2}$ distributions.

A discrete double generalized exponential (DDGE) distribution of Nekoukhou and Bidram (2015a) is a special case of the DBGE distribution, when $a=1$. Moreover, a discrete beta-exponential (DBE) distribution of Nekoukhou and Bidram (2015a) is another sub-model of DBGE distribution, when $\alpha=1$.

(5) The discrete beta-Rayleigh distribution, $\operatorname{DBR}(a, b, p)$, as mentioned in the present paper, is the same as that of $\operatorname{DBW}(a, b, 2, p)$ distribution.

The maximum likelihood method is used to obtain the estimates of the parameters of the proposed new distribution. Comparing the DBW model is performed by using the Akaike information criterion (AIC) and Kolmogrov-Smirnov (K-S) test statistic. It must be mentioned that the parameters have been estimated by means of all data (here $n=100$ ), and the estimated model is written as

$$
\widehat{f_{D B W}}(y ; \hat{a}, \hat{b}, \hat{\alpha}, \hat{p})=\sum_{i=0}^{\infty} \omega_{i}(\hat{a}, \hat{b})\left\{\left[1-e^{-\hat{\beta}(y+1)^{\hat{\alpha}}}\right]^{\hat{a}+i}-\left[1-e^{-\hat{\beta} y^{\hat{\alpha}}}\right]^{\hat{a}+i}\right\},
$$

for $y=0,1,2,3,4,5,6,7,8$. In order to calculate the AIC values, for example for the DBW model, we have

$$
\begin{aligned}
\ell(\hat{a}, \hat{b}, \hat{\alpha}, \hat{p})=\log \prod_{i=1}^{9} P\left(\widehat{Y=} y_{i}\right) & =\log P \widehat{(Y=0)}+\log P \widehat{(Y=1)}+\ldots+\log P \widehat{(Y=8)} \\
& =-21.9043
\end{aligned}
$$

and hence

$$
A I C=2 \times 4-2 \times \ell(\hat{a}, \hat{b}, \hat{\alpha}, \hat{p})=2 \times 4-2 \times(-21.9043)=51.8085 .
$$

Table 4 indicates the fitting computations which consists of the MLEs, AICs and the values of K-S test statistic determined by the fitting models.

According to the AICs and the values of K-S test statistic in Table 4, it seems that the DBW model gives a satisfactory fit for these real data. Figures 4 and 5 indicate the fitted models and corresponding probability-probability ( $p-p)$ plots of the fitted models, 
respectively. As we see, the figures also confirm goodness of fit of the DBW model.

The likelihood ratio (LR) method is used to test the null hypotheses $H_{0}$ : DBE, $H_{0}$ : DBR, $H_{0}$ : EDW, $H_{0}$ : GDR, $H_{0}$ : DGE 2 and $H_{0}$ : DW versus the alternative hypothesis

Table 4: Results for fish catch data.

\begin{tabular}{|c|c|c|c|}
\hline Models & MLEs & $\mathrm{AIC}$ & K-S statistic \\
\hline DBW & $\begin{array}{c}(\hat{a}, \hat{b}, \hat{\alpha}, \hat{p})=(1.8864,1.1613,1.4321,0.8835) \\
\hat{S E}(\hat{a}, \hat{b}, \hat{\alpha}, \hat{p})=(0.3284,0.2686,0.4212,0.4508)\end{array}$ & 51.8085 & 0.1629 \\
\hline DBGE & $\begin{array}{c}(\hat{a}, \hat{b}, \hat{\alpha}, \hat{p})=(1.3680,1.5326,1.3720,0.8877) \\
\hat{S E}(\hat{a}, \hat{b}, \hat{\alpha}, \hat{p})=(0.4112,0.3113,0.4456,0.3618)\end{array}$ & 56.9167 & 0.3786 \\
\hline DBR & $\begin{array}{c}(\hat{a}, \hat{b}, \hat{p})=(1.5364,1.3781,0.9523) \\
\hat{S E}(\hat{a}, \hat{b}, \hat{p})=(0.3981,0.4536,0.3343)\end{array}$ & 52.7182 & 0.3021 \\
\hline EDW & $\begin{array}{c}(\hat{\alpha}, \hat{\gamma}, \hat{p})=(2.7425,1.5681,0.9833) \\
\hat{S E}(\hat{\alpha}, \hat{\gamma}, \hat{p})=(0.2819,0.4336,0.3871)\end{array}$ & 59.6494 & 0.2908 \\
\hline GDR & $\begin{array}{c}(\hat{\gamma}, \hat{p})=(2.8033,0.9249) \\
\hat{S E}(\hat{\gamma}, \hat{p})=(0.2777,0.3029)\end{array}$ & 58.5595 & 0.4135 \\
\hline DBE & $\begin{array}{c}(\hat{a}, \hat{b}, \hat{p})=(1.5431,1.1241,0.9231) \\
\hat{S E}(\hat{a}, \hat{b}, \hat{p})=(0.3642,0.3713,0.2873)\end{array}$ & 62.6518 & 0.6031 \\
\hline DDGE & $\begin{array}{c}(\hat{b}, \hat{\alpha}, \hat{p})=(1.7213,0.9431,0.9463) \\
\hat{S E}(\hat{b}, \hat{\alpha}, \hat{p})=(0.3091,0.3227,0.3335)\end{array}$ & 55.5464 & 0.4702 \\
\hline $\mathrm{DGE}_{2}$ & $\begin{array}{c}(\hat{a}, \hat{p})=(13.7229,0.4969) \\
\hat{S E}(\hat{a}, \hat{p})=(0.1978,0.1793)\end{array}$ & 62.1244 & 0.2565 \\
\hline DW & $\begin{array}{c}(\hat{\alpha}, \hat{p})=(3.5792,0.9969) \\
\hat{S E}(\hat{\alpha}, \hat{p})=(0.2011,0.1834)\end{array}$ & 57.4555 & 0.2960 \\
\hline
\end{tabular}

$H_{1}$ : DBW. The values of LR test statistic and their corresponding $p$-values for the data set are given in Table 5 .

According to the results, we see that the null hypotheses are rejected in favor of the alternative hypothesis at the usual significance level.

One can construct approximate confidence intervals for the parameters of the DBW model. Indeed, such confidence intervals are attained by means of the asymptotic covariance matrix of the MLEs of DBW parameters when the Newton-Raphson procedure converges. For instance, 95\% asymptotic confidence intervals for DBW parameters are calculated as $a \in(1.8864 \pm 0.6437), b \in(1.1613 \pm 0.5264), \alpha \in(1.4321 \pm 0.8255)$ and $p \in(0.8835 \pm 0.1273)$.

In view of the fact that the above confidence intervals are finite, we can conclude that the parameters of the distribution in question are identifiable; see Raue et al. (2009). 
Table 5: LR test for fish catch data.

\begin{tabular}{cccc}
\hline$H_{0}$ & $H_{1}$ & LR value & $p$-value \\
\hline $\operatorname{DBE}(\alpha=1)$ & $\mathrm{DBW}(\alpha \neq 1)$ & 12.8433 & $3.38 \times 10^{-4}(<0.01)$ \\
$\operatorname{DBR}(\alpha=2)$ & $\mathrm{DBW}(\alpha \neq 2)$ & 2.9096 & $0.0881(<0.10)$ \\
$\operatorname{EDW}(b=1)$ & $\mathrm{DBW}(b \neq 1)$ & 9.8408 & $0.0017(<0.01)$ \\
$\operatorname{GDR}(\alpha=2, b=1)$ & $\mathrm{DBW}(\alpha \neq 2, b \neq 1)$ & 10.7510 & $0.0010(<0.01)$ \\
$\operatorname{DGE}_{2}(\alpha=1, b=1)$ & $\mathrm{DBW}(\alpha \neq 1, b \neq 1)$ & 14.3158 & $7.78 \times 10^{-4}(<0.01)$ \\
$\operatorname{DW}(a=1, b=1)$ & $\mathrm{DBW}(a \neq 1, b \neq 1)$ & 9.6470 & $0.0080(<0.01)$ \\
\hline
\end{tabular}

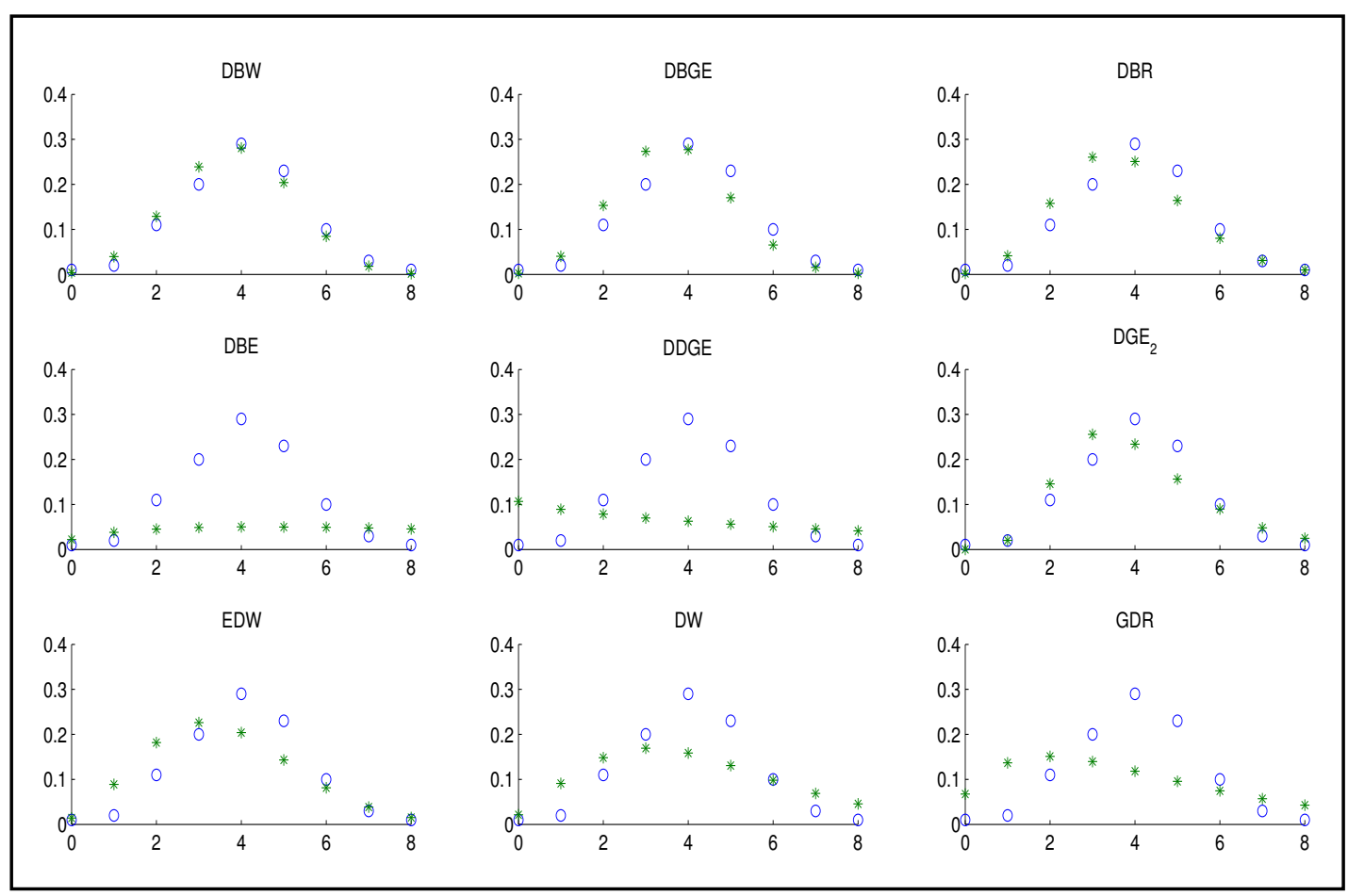

Figure 4: The fitted models for fish catch data.

\section{Conclusions}

We introduced a new discrete distribution, called discrete beta-Weibull (DBW) distribution, motivated by the fact that it provides greater flexibility in order to analyze various discrete data. Indeed, the DBW distribution is appropriate for modeling both over and under-dispersed data and its hazard rate function can be bathtub-shaped, unimodal, increasing and decreasing. That is, DBW distributions can be used as improved models for analyzing failure data in the discrete case.

In this paper we have developed mainly the classical inference. It will be interesting to develop the Bayesian inference also and compare their performances. Moreover, here have considered univariate model, it is important to see how it can be generalized to the 


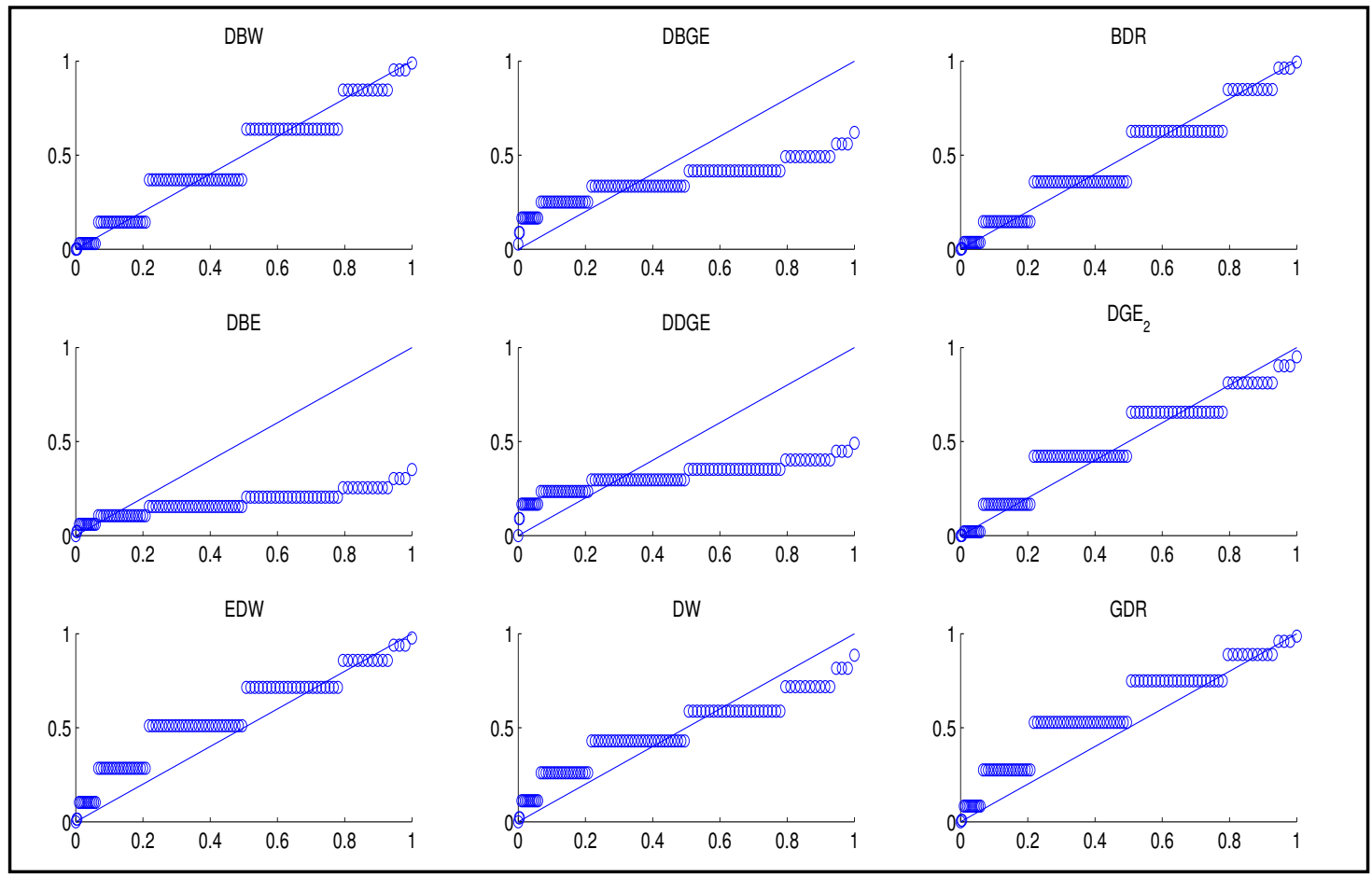

Figure 5: The p-p plots of fitted models for fish catch data.

bivariate and even multivariate case. More work is needed along these directions.

\section{Acknowledgments}

The authors would like to thank two unknown referees for their constructive comments which had helped to improve the manuscript in a significant manner.

\section{References}

[1] Barreto-Souza, W., Santos, A. H., Cordeiro, G.M. (2010). The beta generalized exponential distribution. J. Stat. Comput. Simul.. 80(2): 159-172.

[2] Bidram, H., Behboodian, J., Towhidi, M. (2013). The beta Weibull-geometric Distribution. J. Stat. Comput. Simul., 83(1): 52-67.

[3] Castellares, F., Montenegro, L.C., Cordeiro, G.M. (2013). The beta log-normal distribution. J. Stat. Comput. Simul., 83(2): 203-228.

[4] Chakraborty, S., Chakravarty, D. (2012). Discrete Gamma distributions: properties and parameter estimation. Comm. Stat.Theo. Meth., 41(18): 3301-3324.

[5] Chakraborty, S., Chakravarty, D. (2014). A Discrete Gumbel Distribution. arXiv: 1410.7568.

[6] Cordeiro, G.M., Gomes, A.E., Silva, C.Q., Ortega, E.M.M. (2013a). The beta exponentiated Weibull distribution. J. Stat. Comput. Simul., 83(1): 114-138. 
[7] Cordeiro, G.M., Cristino, C.T., Hashimoto, E.M., Ortega, E.M.M. (2013b). The beta generalized Rayleigh distribution with applications to lifetime data. Stat. Papers, 54: 133-161.

[8] Cordeiro, G.M., Silva, G.O., Ortega, E.M.M. (2013c). The beta-Weibull geometric distribution, Statistics, 47: 817-834.

[9] Cordeiro, G.M., Simas, A.B., Stošić, B.D. (2011). Closed form expressions for moments of the beta Weibull distribution. Anais da Academia Brasileira de Ciências, 83(2): 357-373.

[10] Cordeiro, G.M., Lemonte, A.J. (2011a). The beta Laplace distribution. Stat. \& Prob. Lett., 81: 973982.

[11] Cordeiro, G.M., Lemonte, A.J. (2011b). The beta-half-Cauchy distribution. J. Prob. Stat., 2011: $1-18$.

[12] Eugene, N., Lee, C. Famoye, F. (2002). Beta-normal distribution and its applications. Comm. Stat. Theo. Meth., 31: 497-512.

[13] Ferguson, T.S. (1996). A course in large sample theory. Chapman and Hall, London.

[14] Gómez-Déniz E. (2010). Another generalization of the geometric distribution, Test, 19: 399-415.

[15] Gómez-Déniz E., Calderin-Ojeda, E. (2011). The discrete Lindley distribution: properties and applications. J. Stat. Comput. Simul., 81(11): 1405-1416.

[16] Gupta, R.D., Kundu, D. (1999). Generalized exponential distributions. Aust. NZ. J. Stat., 41(2): 173-188.

[17] Hussain, T., Ahmad, M. (2014). Discrete Inverse Rayleigh Distribution. Pak. J. Statist., 30(2): 203222 .

[18] Inusah, S., Kozubowski, T.J. (2006). A discrete analogue of the Laplace distribution. Statist. Plann. Infe., 136: 1090-1102.

[19] Kemp, A.W. (1992). HeineEuler extensions of the Poisson distribution. Commun. Stat. TheoryMethods, 21(3): 571-588.

[20] Kemp, A.W. (1997). Characterizations of discrete normal distribution. Statist. Plann. Inf., 63: 223229.

[21] Kotz, S. Lumelskii Y., Pensky, M. (2003). The stress-strength model and its generalizations: Theory and Applications. Singapore: World Scientific 43, 44.

[22] Kozubowski, T.J., Inusah, S. (2006). A skew Laplace distribution on integers. AISM, 58: 555-571.

[23] Krishna, H., Pundir, P.S. (2007). Discrete maxwell distribution. InterStat, November.

[24] Krishna, H., Pundir, P.S. (2009). Discrete Burr and discrete Pareto distributions. Statist. Methodol., 6: $177-188$.

[25] Lee, H., Cha, J.H. (2015). On Two General Classes of Discrete Bivariate Distributions. The American Statistician, DOI: 10.1080/00031305.2015.1044564.

[26] Lemonte, A.J., Cordeiro, G.M. (2013). An extended Lomax distribution. Statistics, 47(4): 800-816. bibitem Lisman, J.H.C., van Zuylen, M.C.A. (1972). Note on the generation of most probable frequency distributions. Statistica. Neerlan., 26(1): 19-23. 
[27] Mahmoudi, E. (2011). The beta generalized Pareto distribution with application to lifetime data. Math. Comput. Simul., 81: 2414-2430.

[28] Morais, A.L., Cordeiro, G.M., Audrey, H.M.A. (2013). The beta generalized logistic distribution. Brazilian J. Prob. Stat., 27: 185-200.

[29] Mudholkar, G.S, Srivastava, D.K., Freimer, M. (1995). The exponentiated Weibull family: A reanalysis of the bus-motor-failure data. Technometrics, 37: 436-445.

[30] Nadarajah, S., Kotz, S. (2006). The beta exponential distribution. Reli. Engin. Sys. Saf., 91: 689-697.

[31] Nakagawa, T., Osaki, S. (1975). The discrete Weibull distribution. IEEE Transact. Reliab., 24(5): 300-301.

[32] Nekoukhou, V., Alamatsaz, M.H., Bidram, H. (2013). Discrete generalized exponential distribution of a second type. Statistics, 47(4): 876-887.

[33] Nekoukhou, V., Alamatsaz, M.H. Bidram, H. Aghajani, A.H. (2015). Discerte beta exponential distribution. Commun. Stat. Theory-Methods, 44: 2079-2091.

[34] Nekoukhou, V., Bidram, H. (2015a). A new four-parameter discrete distribution with bathtub and unimodal failur rate. J. Applied Statistics, 42(12): 2654-2670.

[35] Nekoukhou, V., Bidram, H. (2015b). The exponentiated discrete Weibull distribution. SORT, 39(1): 127-146.

[36] Nekoukhou, V. (2015). The Beta-Rayleigh Distribution on the Lattice of Integers. J. Statistical research of Iran, 12: 205224.

[37] Raue, A., Kreutz, C., Maiwald, T., Bachmann, J., Schilling, M., Klingmller, U., Timmer, J. (2009). Structural and practical identifiability analysis of partially observed dynamical models by exploiting the profile likelihood. Bioinformatics, 25(15), 1923-1929.

[38] Roy, D. (2003). The discrete normal distribution. Comm. Stat.Theo. Meth., 32(10): 1871-1883.

[39] Roy, D. (2004). Discrete Rayleigh distribution,. IEEE Trans. Reliab, 53: 255-260.

[40] Silva, G.O., Ortega, E.M.M., Cordeiro, G.M. (2010). The beta modified Weibull distribution. Life. data Anal., 16: 409-430.

[41] Singla, N., Jain, K., Sharma, S.K. (2012). The beta generalized Weibull distribution: Properties and applications. Reli. Engin. Sys. Saf., 102: 5-15.

[42] Steutel, F.W., van Harn, K. (2004). Infinite Divisibility of Probability Distributions on the Real Line. New York: Marcel Dekker. 